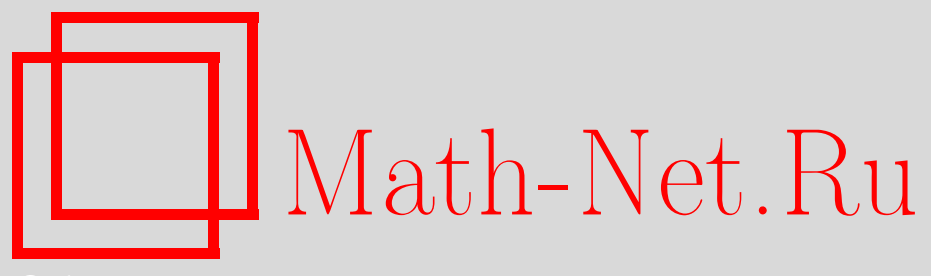

А. Б. Пименов, К. В. Степаньянц, Четырехпетлевая проверка алгоритма суммирования диаграмм Фейнмана в $N=1$ суперсимметричной электродинамике, $T M \Phi$, 2006, том 147, номер 2, 290-302

DOI: https://doi.org/10.4213/tmf1964

Использование Общероссийского математического портала Math-Net.Ru подразумевает, что вы прочитали и согласны с пользовательским соглашением http://www . mathnet.ru/rus/agreement

Параметры загрузки:

IP : 54.166 .219 .16

26 апреля 2023 г., 14:39:55

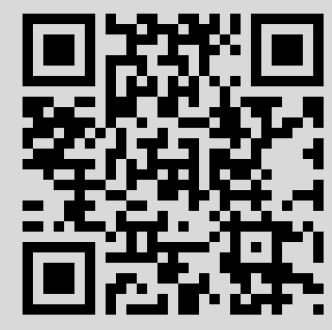




\title{
ЧЕТЫРЕХПЕТЛЕВАЯ ПРОВЕРКА АЛГОРИТМА СУММИРОВАНИЯ ДИАГРАММ ФЕЙНМАНА В $N=1$ СУПЕРСИММЕТРИЧНОЙ ЭЛЕКТРОДИНАМИКЕ
}

\begin{abstract}
С помощью вычисления ряда четырехпетлевых диаграмм в $N=1$ суперсимметричной электродинамике, регуляризованной высшими производными, проверяется метод суммирования диаграмм Фейнмана, основанный на использовании уравнений Швингера-Дайсона и тождеств Уорда. В частности, для рассматриваемых диаграмм доказана справедливость дополнительного тождества для функций Грина, которое не сводится к калибровочному тождеству Уорда.
\end{abstract}

Ключевые слова: суперсимметрия, тождество Уорда, высшие ковариантные производные.

\section{1. ВВЕДЕНИЕ}

Косвенные экспериментальные подтверждения существования суперсимметрии в Стандартной модели делают особенно актуальной проблему вычисления квантовых поправок в суперсимметричных теориях. Благодаря суперсимметрии существенно улучшается ультрафиолетовое поведение моделей теории поля. Например, даже в теориях с нерасширенной суперсимметрией можно сделать предположение о виде $\beta$ функции во всех порядках теории возмущений. В случае $N=1$ суперсимметричной электродинамики, который будет рассмотрен в этой работе, такая $\beta$-функция (называемая точной $\beta$-функцией Новикова-Шифмана-Вайнштейна-Захарова (НШВЗ)) имеет вид [1]

$$
\beta(\alpha)=\frac{\alpha^{2}}{\pi}(1-\gamma(\alpha)),
$$

где $\gamma(\alpha)$ - аномальная размерность суперполя материи.

Явные вычисления, выполненные при использовании регуляризации размерной редукцией [2], подтверждают это предположение, но требуют специального подбора схемы вычитаний [3]. При использовании регуляризации высшими производными [4] явные вычисления в двух- [5] и трехпетлевом [6] приближениях для $N=1$ суперсимметричной электродинамики показали, что перенормировка оператора $W_{a} C^{a b} W_{b}$

* Московский государственный университет, Москва, Россия. E-mail: stepan@phys.msu.ru 
является чисто однопетлевой, а функция Гелл-Манна-Лоу совпадает с точной $\beta$ функцией НШВЗ и имеет поправки во всех порядках теории возмущений.

Попытка провести явные вычисления точно во всех порядках теории возмущений была сделана в работе [7]. В ней было показано, что с помощью уравнений Швингера-Дайсона и тождеств Уорда можно вычислить значительное число диаграмм Фейнмана точно во всех порядках теории возмущений. Тем не менее ряд диаграмм таким образом определить не удалось. Однако явные вычисления (до трех петель включительно) свидетельствуют о том, что неопределенные вклады в сумме оказываются равными нулю. Это подразумевает существование некоторого тождества, которое не сводится к тождествам Уорда и графически может быть записано в виде (31); в аналитическом виде это тождество дается формулой (30) (см. ниже). Оно связывает между собой двухточечную функцию Грина суперполя материи и трехточечную функцию Грина, в которой на внешних линиях стоят калибровочное суперполе, киральное суперполе материи и специальным образом введенный внешний источник, представляющий собой произвольное скалярное суперполе.

Важность тождества (30) заключается в том, что оно нетривиальным образом ограничивает структуру расходимостей теории. Действительно, суперсимметрия и калибровочная инвариантность требуют, чтобы двухточечная функция Грина калибровочного поля была бы пропорциональна $V \partial^{2} \Pi_{1 / 2} V$, причем коэффициент при этом выражении является некоторой неизвестной функцией (это есть суперсимметричный аналог условия поперечности в обычной электродинамике.) Используя уравнения Швингера-Дайсона и тождества Уорда, можно переписать эту функцию через двухточечную функцию Грина суперполей материи и некоторую другую функцию, которая калибровочной инвариантностью не фиксируется. Фактически это означает, что двухточечная функция Грина калибровочного суперполя эквивалентно переписывается в некотором другом виде, по-прежнему с точностью до не определенной (из тождеств Уорда или, эквивалентно, из требования калибровочной инвариантности) функции. Однако новое тождество говорит о том, что те члены, которые зависят от неизвестной функции, на самом деле оказываются нулевыми. Тем самым это тождество действительно ликвидирует произвол в структуре расходимости.

Конечно, крайне желательно было бы найти истинную причину возникновения такого тождества - например, некоторую симметрию. Но прежде всего необходимо убедиться, что оно действительно имеет место, а не связано с некоторым случайным совпадением.

Нетривиальным рассматриваемое тождество становится начиная с трехпетлевого приближения. В работе [8] оно доказано во всех порядках теории возмущений для некоторого специального класса диаграмм. В таких диаграммах имеется только одна петля суперполей материи, причем два произвольных разреза этой петли не делают диаграмму несвязной (в этом случае техническая часть доказательства является более простой). Возможно, в общем случае доказательство может быть проведено аналогичным образом. Чтобы быть уверенным в возможности проведения такого доказательства, желательно проверить тождество (30) для других диаграмм, что требует проведения явных четырехпетлевых вычислений. Эти вычисления выпол- 
няются в данной работе для некоторой группы четырехпетлевых диаграмм, которые могут быть сделаны несвязными двумя разрезами петли суперполей материи.

Статья построена следующим образом. Основные сведения о $N=1$ суперсимметричной электродинамике и ее регуляризации с помощью метода высших производных напоминаются в разделе 2. Способ суммирования диаграмм Фейнмана, основанный на использовании уравнений Швингера-Дайсона и тождеств Уорда, описывается в разделе 3. Там же записано тождество, которому предположительно должны удовлетворять функции Грина. Четырехпетлевая проверка результатов, сформулированных в разделе 3 , осуществляется в разделе 4 . Краткий итог работы подводится в заключении.

\section{2. $N=1$ СУПЕРСИММЕТРИЧНАЯ ЭЛЕКТРОДИНАМИКА И РЕГУЛЯРИЗАЦИЯ ВЫСШИМИ ПРОИЗВОДНЫМИ}

Безмассовая $N=1$ суперсимметричная электродинамика при наличии слагаемого с высшими производными в суперпространстве описывается следующим действием:

$$
S=\frac{1}{4 e^{2}} \operatorname{Re} \int d^{4} x d^{2} \theta W_{a} C^{a b}\left(1+\frac{\partial^{2 n}}{\Lambda^{2 n}}\right) W_{b}+\frac{1}{4} \int d^{4} x d^{4} \theta\left(\phi^{*} e^{2 V} \phi+\tilde{\phi}^{*} e^{-2 V} \tilde{\phi}\right) .
$$

При этом $\phi$ и $\tilde{\phi}$ - киральные суперполя материи, а $V$ - вещественное скалярное суперполе, которое в качестве одной из компонент содержит калибровочное поле $A_{\mu}$. Суперполе $W_{a}$ представляет собой суперсимметричный аналог тензора напряженности калибровочного поля и в абелевом случае определяется как

$$
W_{a}=\frac{1}{16} \bar{D}\left(1-\gamma_{5}\right) D\left[\left(1+\gamma_{5}\right) D_{a} V\right]
$$

где $D$ - суперсимметричная ковариантная производная. Важно отметить, что в абелевом случае суперполе $W^{a}$ является калибровочно-инвариантным, благодаря чему действие (2) также будет калибровочно-инвариантным.

Квантование модели (2) может быть выполнено стандартным образом. Для этого удобно использовать технику суперграфов, подробно описанную в книге [9], и фиксировать калибровку добавлением следующих слагаемых:

$$
S_{\mathrm{gf}}=-\frac{1}{64 e^{2}} \int d^{4} x d^{4} \theta\left(V D^{2} \bar{D}^{2}\left(1+\frac{\partial^{2 n}}{\Lambda^{2 n}}\right) V+V \bar{D}^{2} D^{2}\left(1+\frac{\partial^{2 n}}{\Lambda^{2 n}}\right) V\right),
$$

где

$$
D^{2} \equiv \frac{1}{2} \bar{D}\left(1+\gamma_{5}\right) D, \quad \bar{D}^{2} \equiv \frac{1}{2} \bar{D}\left(1-\gamma_{5}\right) D .
$$

После добавления таких членов квадратичная по суперполю $V$ часть действия будет иметь наиболее простой вид:

$$
S_{\text {gauge }}+S_{\text {gf }}=\frac{1}{4 e^{2}} \int d^{4} x d^{4} \theta V \partial^{2}\left(1+\frac{\partial^{2 n}}{\Lambda^{2 n}}\right) V .
$$

В рассматриваемом здесь абелевом случае диаграммы, содержащие духовые петли, отсутствуют. 
Хорошо известно, что введение слагаемого с высшими производными не устраняет расходимости в однопетлевых диаграммах. Для их регуляризации необходимо вставить в производящий функционал детерминанты полей Паули-Вилларса [10].

Производящий функционал можно записать в виде

$$
Z=\int D V D \phi D \tilde{\phi} \prod_{i}\left(\operatorname{det} \operatorname{PV}\left(V, M_{i}\right)\right)^{c_{i}} \exp \left(i\left(S_{\text {ren }}+S_{\text {gf }}+S_{\mathrm{S}}+S_{\phi_{0}}\right)\right) \text {. }
$$

При этом

$$
\begin{gathered}
S_{\text {ren }}=\frac{1}{4 e^{2}} Z_{3}\left(e, \frac{\Lambda}{\mu}\right) \operatorname{Re} \int d^{4} x d^{2} \theta W_{a} C^{a b}\left(1+\frac{\partial^{2 n}}{\Lambda^{2 n}}\right) W_{b}+ \\
+Z\left(e, \frac{\Lambda}{\mu}\right) \frac{1}{4} \int d^{4} x d^{4} \theta\left(\phi^{*} e^{2 V} \phi+\tilde{\phi}^{*} e^{-2 V} \tilde{\phi}\right)
\end{gathered}
$$

- перенормированное действие; действие для членов, фиксирующих калибровку, дается формулой (4) (в нем удобно заменить $e$ на голый заряд $e_{0}$ ); детерминанты Паули-Вилларса определяются как

$$
(\operatorname{det} \operatorname{PV}(V, M))^{-1}=\int D \Phi D \widetilde{\Phi} \exp \left(i S_{\mathrm{PV}}\right),
$$

где

$$
\begin{aligned}
S_{\mathrm{PV}} \equiv Z & \left(e, \frac{\Lambda}{\mu}\right) \frac{1}{4} \int d^{4} x d^{4} \theta\left(\Phi^{*} e^{2 V} \Phi+\widetilde{\Phi}^{*} e^{-2 V} \widetilde{\Phi}\right)+ \\
& +\frac{1}{2} \int d^{4} x d^{2} \theta M \widetilde{\Phi} \Phi+\frac{1}{2} \int d^{4} x d^{2} \bar{\theta} M \widetilde{\Phi}^{*} \Phi^{*}
\end{aligned}
$$

а коэффициенты $c_{i}$ удовлетворяют условиям

$$
\sum_{i} c_{i}=1, \quad \sum_{i} c_{i} M_{i}^{2}=0
$$

Далее мы будем предполагать, что $M_{i}=a_{i} \Lambda$, где $a_{i}$ - некоторые постоянные. Вставка детерминантов Паули-Вилларса позволяет сократить остаточные расходимости во всех однопетлевых диаграммах, в том числе в диаграммах, содержащих контрчленные вставки.

Члены с источниками записываются в виде

$$
S_{\mathrm{S}}=\int d^{4} x d^{4} \theta J V+\int d^{4} x d^{2} \theta(j \phi+\tilde{j} \tilde{\phi})+\int d^{4} x d^{2} \bar{\theta}\left(j^{*} \phi^{*}+\tilde{j}^{*} \tilde{\phi}^{*}\right) .
$$

Кроме того, в производящий функционал (7) мы ввели выражение

$$
S_{\phi_{0}}=\frac{1}{4} \int d^{4} x d^{4} \theta\left(\phi_{0}^{*} e^{2 V} \phi+\phi^{*} e^{2 V} \phi_{0}+\tilde{\phi}_{0}^{*} e^{-2 V} \tilde{\phi}+\tilde{\phi}^{*} e^{-2 V} \tilde{\phi}_{0}\right),
$$

где $\phi_{0}, \phi_{0}^{*}, \quad \tilde{\phi}_{0}$ и $\tilde{\phi}_{0}^{*}-$ некоторые скалярные суперполя, которые не являются киральными или антикиральными и представляют собой некоторые параметры. Вообще говоря, вводить слагаемое $S_{\phi_{0}}$ в производящий функционал не обязательно, 
однако присутствие параметров $\phi_{0}$ крайне желательно при исследовании уравнений Швингера-Дайсона.

В наших обозначениях производящий функционал для связных функций Грина записывается в виде

$$
W=-i \ln Z,
$$

а эффективное действие получается с помощью преобразования Лежандра:

$$
\Gamma=W-\int d^{4} x d^{4} \theta J V-\int d^{4} x d^{2} \theta(j \phi+\tilde{j} \tilde{\phi})-\int d^{4} x d^{2} \bar{\theta}\left(j^{*} \phi^{*}+\tilde{j}^{*} \tilde{\phi}^{*}\right),
$$

причем источники $J, j$ и $\tilde{j}$ должны быть выражены через поля $V, \phi$ и $\tilde{\phi}$ с помощью уравнений

$$
V=\frac{\delta W}{\delta J}, \quad \phi=\frac{\delta W}{\delta j}, \quad \tilde{\phi}=\frac{\delta W}{\delta \tilde{j}} .
$$

\section{3. СУММИРОВАНИЕ ДИАГРАММ ФЕЙНМАНА В $N=1$ СУПЕРСИММЕТРИЧНОЙ КВАНТОВОЙ ЭЛЕКТРОДИНАМИКЕ}

Из производящего функционала (7) можно получить [7] уравнения ШвингераДайсона, которые графически записываются в виде
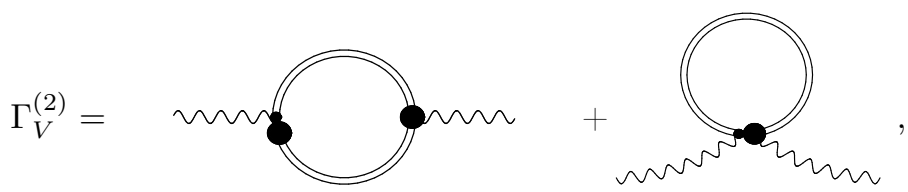

где $\Gamma_{V}^{(2)}$ - двухточечная функция Грина калибровочного поля. При этом мы везде будем полагать константу перенормировки $Z=1$, поскольку зависимость от $Z$ может быть легко восстановлена в окончательном результате и эта зависимость не будет существенна в данной работе.

В формуле (17) двойная линия обозначает точный пропагатор $(Z=1)$

$$
\left(\frac{\delta^{2} \Gamma}{\delta \phi_{x}^{*} \delta \phi_{y}}\right)^{-1}=-\frac{D_{x}^{2} \bar{D}_{x}^{2}}{4 \partial^{2} G} \delta_{x y}^{8},
$$

в котором функция $G\left(q^{2}\right)$ определяется по двухточечной функции Грина следующим образом:

$$
\frac{\delta^{2} \Gamma}{\delta \phi_{x}^{*} \delta \phi_{y}}=\frac{D_{x}^{2} \bar{D}_{x}^{2}}{16} G\left(\partial^{2}\right) \delta_{x y}^{8},
$$

где $\delta_{x y}^{8} \equiv \delta^{4}(x-y) \delta^{4}\left(\theta_{x}-\theta_{y}\right)$, а индексы внизу указывают точки, в которых берутся рассматриваемые величины.

Большой круг обозначает эффективную вершину, которая в силу тождеств Уорда записывается в виде [7]

$$
\begin{aligned}
\left.\frac{\delta^{3} \Gamma}{\delta V_{x} \delta \phi_{y} \delta \phi_{z}^{*}}\right|_{p=0}= & \partial^{2}\left(\Pi_{1 / 2}\right)_{x}\left(\bar{D}_{x}^{2} \delta_{x y}^{8} D_{x}^{2} \delta_{x z}^{8}\right) F\left(q^{2}\right)+ \\
& +\frac{1}{32} q^{\mu} G^{\prime}\left(q^{2}\right) \bar{D} \gamma^{\mu} \gamma_{5} D_{x}\left(\bar{D}_{x}^{2} \delta_{x y}^{8} D_{x}^{2} \delta_{x z}^{8}\right)+\frac{1}{8} \bar{D}_{x}^{2} \delta_{x y}^{8} D_{x}^{2} \delta_{x z}^{8} G\left(q^{2}\right)
\end{aligned}
$$


где штрихи обозначают производные по $q^{2}$,

$$
\Pi_{1 / 2}=-\frac{1}{16 \partial^{2}} D^{a} \bar{D}^{2} D_{a}=-\frac{1}{16 \partial^{2}} \bar{D}^{a} D^{2} \bar{D}_{a}
$$

- суперсимметричный поперечный проектор, а $F\left(q^{2}\right)$ представляет собой некоторую функцию, которая не может быть определена из тождеств Уорда. При этом

$$
\begin{aligned}
D^{a} & \equiv\left[\frac{1}{2} \bar{D}\left(1+\gamma_{5}\right)\right]^{a}, & D_{a} & \equiv\left[\frac{1}{2}\left(1+\gamma_{5}\right) D\right]_{a}, \\
\bar{D}^{a} & \equiv\left[\frac{1}{2} \bar{D}\left(1-\gamma_{5}\right)\right]^{a}, & \bar{D}_{a} & \equiv\left[\frac{1}{2}\left(1-\gamma_{5}\right) D\right]_{a} .
\end{aligned}
$$

Два смежных круга представляют собой эффективную вершину, состоящую из одночастично неприводимых диаграмм, в которых одна из внешних линий прикреплена к самому левому краю. Такие вершины даются выражениями [7]

$$
\frac{\delta^{2} \Gamma}{\delta \phi_{y} \delta \phi_{0 z}^{*}}=\frac{1}{4} \frac{\delta}{\delta \phi_{y}} \exp \left(\frac{2}{i} \frac{\delta}{\delta J_{z}}+2 V_{z}\right) \phi_{z}=-\frac{1}{8} G \bar{D}_{y}^{2} \delta_{y z}^{8}
$$

в случае одной внешней $V$-линии (вершина в первой диаграмме уравнения (17)) и

$$
\begin{aligned}
\left.\frac{\delta^{3} \Gamma}{\delta V_{x} \delta \phi_{y} \delta \phi_{0 z}^{*}}\right|_{p=0}= & \left.\frac{1}{4} \frac{\delta}{\delta V_{x}} \frac{\delta}{\delta \phi_{y}} \exp \left(\frac{2}{i} \frac{\delta}{\delta J_{z}}+2 V_{z}\right) \phi_{z}\right|_{p=0}= \\
= & -2 \partial^{2}\left(\Pi_{1 / 2}\right)_{x}\left(\bar{D}_{x}^{2} \delta_{x y}^{8} \delta_{x z}^{8}\right) F\left(q^{2}\right)+\frac{1}{8} D^{a} C_{a b} \bar{D}_{x}^{2}\left(\bar{D}_{x}^{2} \delta_{x y}^{8} D_{x}^{b} \delta_{x z}^{8}\right) f\left(q^{2}\right)- \\
& \quad-\frac{1}{16} q^{\mu} G^{\prime}\left(q^{2}\right) \bar{D} \gamma^{\mu} \gamma_{5} D_{x}\left(\bar{D}_{x}^{2} \delta_{x y}^{8} \delta_{x z}^{8}\right)-\frac{1}{4} \bar{D}_{x}^{2} \delta_{x y}^{8} \delta_{x z}^{8} G\left(q^{2}\right)
\end{aligned}
$$

для случая двух внешних $V$-линий (во второй диаграмме уравнения (17)). При этом $f\left(q^{2}\right)$ представляет собой еще одну функцию, которая не может быть определена из тождества Уорда.

Величина

$$
\left.\frac{d}{d \ln \Lambda} \Gamma_{V}^{(2)}\right|_{p=0}
$$

может быть вычислена [7] при помощи подстановки решений тождеств Уорда в уравнения Швингера-Дайсона.

Результат вычисления любой диаграммы может быть записан в виде

$$
\int \frac{d^{4} p}{(2 \pi)^{4}} d^{4} \theta\left(V \partial^{2} \Pi_{1 / 2} V A\left(p^{2}\right)+V^{2} B\left(p^{2}\right)\right),
$$

где $A$ и $B$ - некоторые функции. Это фактически является (с небольшой модификацией) суперсимметричным аналогом разложения на продольную и поперечную части. В силу тождества Уорда в сумме диаграмм должны остаться только поперечные части, т.е. члены, пропорциональные $V \partial^{2} \Pi_{1 / 2} V$. Это подтверждается и вычислениями, сделанными в работе [7]: члены, пропорциональные $V^{2}$, равны нулю 
по сумме диаграмм, и мы не будем их выписывать. Члены же, пропорциональные $V \partial^{2} \Pi_{1 / 2} V$, в эффективных диаграммах оказываются равными

$$
=V \partial^{2} \Pi_{1 / 2} V \frac{d}{d \ln \Lambda} \int \frac{d^{4} q}{(2 \pi)^{4}}\left(\frac{8 F}{q^{2} G}+\frac{1}{2 q^{2}} \frac{d}{d q^{2}} \ln \left(q^{2} G^{2}\right)\right) \text {, }
$$

(здесь мы опустили интегрирование по внешнему импульсу и внешнему $\theta$ ). В выражениях для этих диаграмм также присутствуют члены, пропорциональные $V^{2}$, и аналогичные члены с полями Паули-Вилларса, которые мы для краткости не выписали. Выражения для них можно найти в работе [7]. Члены с полями ПаулиВилларса дают дополнительные вклады, которые сокращают остаточные расходимости. Их исследование технически немного более сложно и требует проведения аналогичных вычислений в массивной теории. Мы всегда будем подразумевать наличие членов с полями Паули-Вилларса, однако явно выписывать их не будем.

Заметим теперь, что при суммировании вкладов (27) и (28) слагаемые, содержащие неизвестную функцию $F$, полностью сокращаются. Однако остаются слагаемые, содержащие неизвестную функцию $f$ :

$$
\left.\frac{d}{d \ln \Lambda} \frac{\delta^{2} \Delta \Gamma}{\delta V_{x} \delta V_{y}}\right|_{p=0}=2 \partial^{2} \Pi_{1 / 2} \delta_{x y}^{8} \int \frac{d^{4} q}{(2 \pi)^{4}}\left(\frac{1}{2 q^{2}} \frac{d}{d q^{2}} \ln \left(q^{2} G^{2}\right)-\frac{8 f}{q^{2} G}-(\mathrm{PV})\right)
$$

где через (PV) обозначены аналогичные члены с полями Паули-Вилларса. Тем не менее явные трехпетлевые вычисления указывают на существование тождества

$$
\frac{d}{d \ln \Lambda} \int \frac{d^{4} q}{(2 \pi)^{4}} \frac{f\left(q^{2}\right)}{q^{2} G\left(q^{2}\right)}=0
$$

где функция $G$ определена по двухточечной функции Грина суперполя материи формулой (19), а функция $f$ - по трехточечной функции Грина с внешними линиями $V, \phi$ и $\phi_{0}^{*}$ формулой (24).

В работе [8] была сделана попытка доказать тождество (30) точно во всех порядках приблизительно тем же методом, с помощью которого производится подиаграммное доказательство тождества Уорда. Несмотря на то что применение такого метода оказалось возможным, из-за технических трудностей доказательство было проведено не в общем случае, а только для диаграмм, которые содержат одну петлю суперполей материи и остаются связными после любых двух разрезов этой петли. В произвольном случае доказательство тем же способом, по-видимому, возможно, однако сопряжено с намного бо́льшими техническими проблемами. 
Также в работе [8] было показано, что графически тождество (30) может быть записано в виде

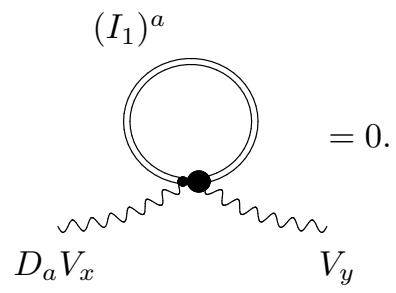

При этом символ $\left(I_{1}\right)^{a}$ (здесь используются обозначения работы [8]) означает, что двойная линия в этом случае соответствует не эффективному пропагатору (18), который пропорционален $D_{x}^{2} \bar{D}_{x}^{2} \delta_{x z}^{8}$, а выражению

$$
-\frac{1}{2 \partial^{2} G\left(\partial^{2}\right)} D_{x}^{a} \bar{D}_{x}^{2} \delta_{x z}^{8} .
$$

Заметим, что равенство (30) не применимо в массивном случае. Соответствующая его модификация может быть найдена в работе [7].

\section{4. ЧЕТЫРЕХПЕТЛЕВЫЕ ВЫЧИСЛЕНИЯ}

Заметим теперь, что выражения (27) и (28) позволяют находить не только суммы всех диаграмм с двумя внешними линиями калибровочного поля, но и суммы определенных классов таких диаграмм. Такие классы диаграмм получаются из некоторого остова, к которому всеми возможными способами прикреплены внешние линии. Так, например, на рис. 1 показан остов для диаграмм, которые исследуются в данной работе, а точками отмечены все возможные места, к которым могут прикрепляться две внешние линии калибровочного поля.

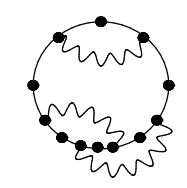

Рис. 1. Схематическое изображение класса диаграмм, исследуемого в работе.

Если остов содержит только одну петлю суперполя материи и не может быть сделан несвязным двумя разрезами этой петли, то для соответствующего класса диаграмм тождество (31) доказано в работе [8]. Однако желательно проверить, будет ли это тождество иметь место и в других случаях. Для этого мы и рассматриваем диаграммы, которые получаются из остова, показанного на рис. 1, добавлением двух внешних линий (очевидно, что такие диаграммы могут быть сделаны несвязными двумя разрезами петли суперполей материи).

Мы будем вычислять рассматриваемые диаграммы двумя способами:

1) по формулам (27) и (28) на основе предварительно полученных функций $G, f$ и $F$;

2) явным вычислением с помощью метода суперграфов. 
Тем самым можно осуществить четырехпетлевую проверку формул (27) и (28), а также тождества (30).

Для того чтобы выяснить, какие диаграммы будут существенны для нахождения неизвестных функций $G, f$ и $F$ в рассматриваемом нами случае, удобно воспользоваться следующими простыми рассуждениями. Диаграмму, изображенную на рис. 1, можно рассматривать как формальное произведение одно- и двухпетлевой диаграмм с внешними линиями $\phi^{*}$ и $\phi$ с попарно отождествленными концами:

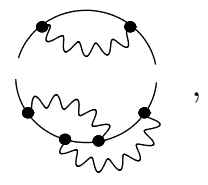

или как трехпетлевую диаграмму, замкнутую саму на себя:
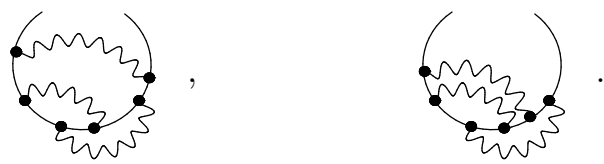

Полученные таким образом части диаграммы и представляют собой диаграммы Фейнмана для определения функции $G$. Для определения функций $f$ и $F$ к таким диаграммам необходимо добавить еще одну внешнюю линию суперполя $V$.

Таким образом, в рассматриваемом случае для нахождения функции $G$ необходимо вычислить диаграммы, изображенные на рис. 2. Сама функция $G$ при этом получается в соответствии с определением (19). Одно-, двух- и трехпетлевую части функции $G$, определяемые диаграммами на рис. 2 , будем обозначать через $G_{1}, G_{2}$ и $G_{3}$, соответственно. Выражения для них приведены в приложении. Полная функция $G$ (конечно, без учета диаграмм, которые не существенны для данной работы) в рассматриваемом приближении равна

$$
G\left(q^{2}\right)=1+G_{1}+G_{2}+G_{3},
$$

где единица представляет собой древесный вклад.
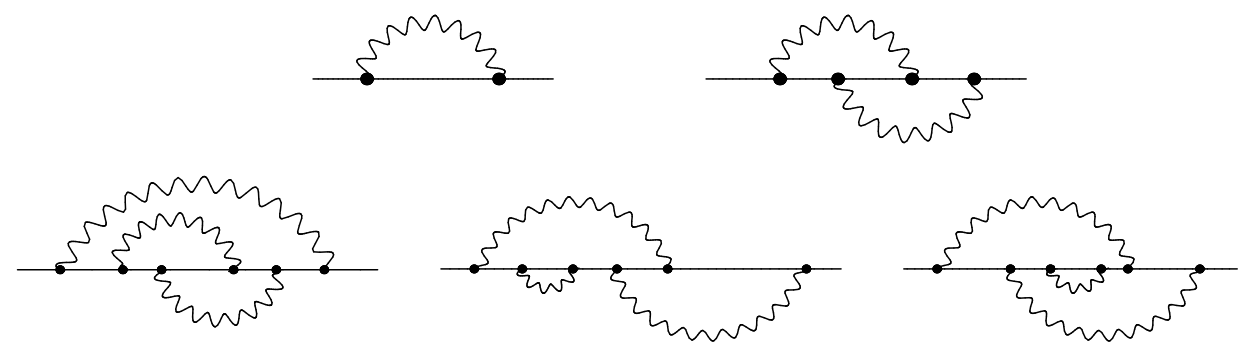

Рис. 2. Диаграммы, используемые для вычисления функции $G$. 
Функции $F\left(q^{2}\right)$ и $f\left(q^{2}\right)$ определяются из трехточечных функций Грина по формуле (24). При этом, как и в случае функции $G$, достаточно вычислить только диаграммы определенного вида, чтобы получить именно тот вклад, который соответствует рассматриваемому классу четырехпетлевых диаграмм. Эти диаграммы получаются из диаграмм, показанных на рис. 2, всевозможными вставками одной внешней $V$-линии.

Одно-, двух- и трехпетлевые вклады в функцию $f$ будем обозначать как $f_{1}, f_{2}$ и $f_{3}$, соответственно. Аналогичные обозначения используем и для функции $F$. Поскольку в древесном приближении эти функции равны нулю и $f_{1}=0$, то в рассматриваемом приближении

$$
F=F_{1}+F_{2}+F_{3}, \quad f=f_{2}+f_{3} .
$$

Выражения для $f_{1}, f_{2}, f_{3}, F_{1}$ и $F_{2}$, полученные в результате вычисления указанных диаграмм, приведены в приложении. Выражение для $F_{3}$ было вычислено, однако не приводится в силу большого размера (для проверки тождества (30) оно не требуется).

Полученные выражения позволяют осуществить проверку тождества (30). С рассматриваемой точностью

$$
\frac{f}{q^{2} G}=\frac{1}{q^{2}}\left(f_{3}-f_{2} G_{1}\right) .
$$

Подставляя сюда выражения для функций $G_{1}, f_{2}$, и $f_{3}$ из равенств (П.1), (П.5), (П.6) (см. приложение), получаем, что подынтегральное выражение можно записать в виде полной производной по импульсу $q$ :

$$
\begin{gathered}
\int \frac{d^{4} q}{(2 \pi)^{4}} \frac{f}{q^{2} G}=i e^{6} \int \frac{d^{4} q d^{4} k d^{4} l d^{4} r}{(2 \pi)^{16}}\left[1+(-1)^{n} \frac{k^{2 n}}{\Lambda^{2 n}}\right]^{-1}\left[1+(-1)^{n} \frac{l^{2 n}}{\Lambda^{2 n}}\right]^{-1} \times \\
\times\left[1+(-1)^{n} \frac{r^{2 n}}{\Lambda^{2 n}}\right]^{-1} \frac{\partial}{\partial q^{\mu}}\left\{\frac{(2 q+k+l)^{\mu}}{k^{2} l^{2} r^{2} q^{2}(k+q)^{2}(q+l)^{2}(q+r)^{2}(k+q+l)^{2}}\right\} .
\end{gathered}
$$

В справедливости этого равенства можно убедиться, если вычислить производную по $q^{\mu}$ при помощи правила Лейбница и сравнить результат с выражением (35), в котором выражения для функций $G_{1}, f_{2}$, и $f_{3}$ получаются с помощью явного вычисления диаграмм.

Поскольку подынтегральное выражение в формуле (36) представляет собой полную производную по импульсу $q^{\mu}$ от выражения, которое стремится к нулю в пределе $q \rightarrow \infty$, то само выражение (36) оказывается равным нулю. Это и означает справедливость тождества (30) в рассматриваемом приближении и для рассматриваемого класса диаграмм.

Заметим, что в данном случае наличие регуляризации с помощью высших производных является весьма существенным. Несмотря на то что параметр $\Lambda$ присутствует только в пропагаторах суперполя $V$, которые не содержат импульса $q$, его наличие позволяет проводить вычисления в пределе $p \rightarrow 0$. Это связано с существованием предела (25), которое доказано в работе [7]. Без использования регуляризации высшими производными переход к этому пределу является бессмысленным, поскольку приводит к появлению бесконечностей. Другая не менее важная причина использования регуляризации высшими производными заключается в том, что в $D \neq 4$ 


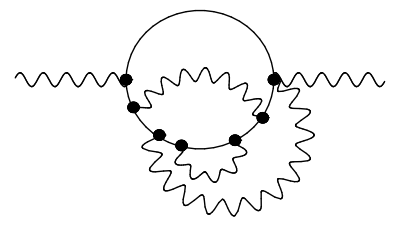

Рис. 3. Одна из диаграмм, дающих вклад в обе эффективные диаграммы.

измерениях могут возникать проблемы с выполнением интегрирования по импульсу $q$ в формуле (29). Например, в $D$ измерениях выражение (35) может не быть представимым в виде полной производной. В принципе можно считать, что тождество (30) есть формальное соотношение в нерегуляризованной теории, которому придается смысл с помощью регуляризации. Однако, по-видимому, в регуляризованной теории оно будет справедливо далеко не для всякой регуляризации. А поскольку в суперсимметричных теориях, как правило, реально используются либо размерная редукция, либо регуляризация высшими производными, то имеет прямой смысл говорить о том, что тождество (30) справедливо в теории, регуляризованной высшими производными.

Желательно также проверить и сам способ суммирования диаграмм Фейнмана с помощью уравнений Швингера-Дайсона и тождеств Уорда. Для этого можно явно вычислить обе эффективные диаграммы в уравнении (17) в четырехпетлевом приближении и сравнить результат с формулами (27) и (28). При этом в рассматриваемом приближении

$$
\begin{gathered}
\frac{1}{q^{2}} \frac{d}{d q^{2}} \ln \left(G^{2}\right)=\frac{q^{\mu}}{q^{4}} \frac{\partial}{\partial q^{\mu}}\left(G_{3}-G_{1} G_{2}\right), \\
\frac{F}{q^{2} G}=\frac{1}{q^{2}}\left(F_{3}-F_{2} G_{1}-F_{1} G_{2}\right) .
\end{gathered}
$$

Явные вычисления проводились с помощью метода, предложенного в работе [11], который упрощает нахождение части диаграммы, пропорциональной $V \partial^{2} \Pi_{1 / 2} V$, но не позволяет получить часть, пропорциональную $V^{2}$. Поэтому проверка формул (27) и (28) проводилась только для части, пропорциональной $V \partial^{2} \Pi_{1 / 2} V$. В обоих случаях она полностью подтвердила их справедливость.

В качестве небольшого технического замечания отметим, что при проведении проверки нужно было учитывать, что многие из обычных диаграмм Фейнмана дают вклады как в первую эффективную диаграмму в уравнении (17), так и во вторую. Например, несложно убедиться, что вклад диаграммы, изображенной на рис. 3, делится на части $3 / 4$ и $1 / 4$, которые соответствуют первой и второй диаграммам в уравнении (17).

\section{5. ЗАКЛЮЧЕНИЕ}

В данной работе была выполнена четырехпетлевая проверка тождества (30) для некоторого класса диаграмм Фейнмана. Для таких диаграмм доказательство, приведенное в работе [8], не применимо, поскольку для них структура суммы диаграмм несколько более сложная и существуют дополнительные ограничения на возможные 
положения внешних линий калибровочного поля. Поэтому полученное здесь подтверждение справедливости рассматриваемого тождества еще раз свидетельствует в пользу того, что оно верно и для произвольных диаграмм. По-видимому, его источником является некоторая симметрия теории. Однако эта симметрия заведомо не есть калибровочная инвариантность.

В заключение еще раз подчеркнем, что особенная важность полученного тождества состоит в том, что оно существенно ограничивает вид расходимостей в рассматриваемой теории. Это связано с тем, что, используя только калибровочную инвариантность, можно свести выражение для двухточечной функции Грина калибровочного поля к выражению, зависящему только от двухточечной функции Грина суперполя материи и левой части этого тождества. Тем самым рассматриваемое тождество удаляет все неопределенные выражения из двухточечной функции Грина калибровочного поля и связывает ее с двухточечной функцией Грина для суперполя материи.

\section{ПРИЛОЖКЕНИЕ}

\section{Явные выражения для функций $G, f$ и $F$}

Ниже приводятся выражения для функций $G, f$ и $F$. При этом индекс указывает, в каком порядке теории возмущений (для диаграмм рассматриваемого класса) была вычислена соответствующая функция.

$$
\begin{aligned}
& G_{1}=2 i e^{2} \int \frac{d^{4} k}{(2 \pi)^{4}}\left[1+(-1)^{n} \frac{k^{2 n}}{\Lambda^{2 n}}\right]^{-1} \frac{1}{k^{2}(q+k)^{2}}, \\
& G_{2}=4 e^{4} \int \frac{d^{4} k d^{4} l}{(2 \pi)^{8}}\left[1+(-1)^{n} \frac{k^{2 n}}{\Lambda^{2 n}}\right]^{-1}\left[1+(-1)^{n} \frac{l^{2 n}}{\Lambda^{2 n}}\right]^{-1} \times \\
& \times \frac{(2 q+k+l)^{2}}{k^{2} l^{2}(k+q)^{2}(q+l)^{2}(k+q+l)^{2}}, \\
& G_{3}=-4 i e^{6} \int \frac{d^{4} k d^{4} l d^{4} r}{(2 \pi)^{12}}\left[1+(-1)^{n} \frac{k^{2 n}}{\Lambda^{2 n}}\right]^{-1}\left[1+(-1)^{n} \frac{l^{2 n}}{\Lambda^{2 n}}\right]^{-1} \times \\
& \times\left[1+(-1)^{n} \frac{r^{2 n}}{\Lambda^{2 n}}\right]^{-1} \frac{1}{k^{2} l^{2} r^{2}(q+k)^{2}(q+l)^{2}(q+k+l)^{2}} \times \\
& \times\left(\frac{(2 q+2 k+r+l)^{2}(q+l)^{2}}{(q+r+k)^{2}(q+r+k+l)^{2}}+\frac{(2 q+k+l)^{2}}{(q+r+k+l)^{2}}+\frac{2(2 q+k+l)^{2}}{(q+r+k)^{2}}\right) ; \\
& f_{1}\left(q^{2}\right)=0 \text {, } \\
& f_{2}\left(q^{2}\right)=\frac{1}{2} e^{4} \int \frac{d^{4} k d^{4} l}{(2 \pi)^{8}}\left[1+(-1)^{n} \frac{k^{2 n}}{\Lambda^{2 n}}\right]^{-1}\left[1+(-1)^{n} \frac{l^{2 n}}{\Lambda^{2 n}}\right]^{-1} \times \\
& \times \frac{1}{k^{2} l^{2}(k+q)^{2}(q+l)^{2}(k+q+l)^{2}} \times \\
& \times\left(-2+\frac{(2 q+k+l)_{\mu}(k+q)^{\mu}}{(k+q)^{2}}+\frac{(2 q+k+l)_{\mu}(l+q)^{\mu}}{(l+q)^{2}}\right), \\
& f_{3}\left(q^{2}\right)=-4 i e^{6} \int \frac{d^{4} k d^{4} l d^{4} r}{(2 \pi)^{12}}\left[1+(-1)^{n} \frac{k^{2 n}}{\Lambda^{2 n}}\right]^{-1}\left[1+(-1)^{n} \frac{l^{2 n}}{\Lambda^{2 n}}\right]^{-1} \times
\end{aligned}
$$




$$
\begin{aligned}
& \times\left[1+(-1)^{n} \frac{r^{2 n}}{\Lambda^{2 n}}\right]^{-1} \frac{1}{k^{2} l^{2} r^{2}(k+q)^{2}(q+l)^{2}(q+k+l)^{2}} \times \\
& \times\left(\frac{1}{(k+q+r)^{2}}+\frac{1}{2(k+l+q+r)^{2}}-\right. \\
& -\frac{(2 q+k+l)^{\mu}(k+q)^{\mu}}{2(k+q)^{2}(k+q+r)^{2}}-\frac{(2 q+k+l)^{\mu}(l+q)^{\mu}}{2(l+q)^{2}(k+q+r)^{2}}- \\
& \left.-\frac{(2 q+k+l)^{\mu}(k+q)^{\mu}}{2(k+q)^{2}(k+l+q+r)^{2}}-\frac{(2 q+k+l)^{\mu}(k+q+r)^{\mu}}{2(k+q+r)^{4}}\right) ; \\
F_{1}\left(q^{2}\right)= & -\frac{i e^{2}}{8} \int \frac{d^{4} k}{(2 \pi)^{4}}\left[1+(-1)^{n} \frac{k^{2 n}}{\Lambda^{2 n}}\right]^{-1} \frac{1}{k^{2}(k+q)^{4}}, \\
F_{2}\left(q^{2}\right)= & \frac{1}{4} e^{4} \int \frac{d^{4} k d^{4} l}{(2 \pi)^{8}}\left[1+(-1)^{n} \frac{k^{2 n}}{\Lambda^{2 n}}\right]^{-1}\left[1+(-1)^{n} \frac{l^{2 n}}{\Lambda^{2 n}}\right]^{-1} \times \\
& \times \frac{(q+l)^{2}}{k^{2} l^{2}(k+q)^{2}(q+l)^{2}(k+q+l)^{2}}\left(4-2 \frac{(2 q+k+l)^{2}}{(k+q)^{2}}\right)
\end{aligned}
$$

(выражение для $F_{3}$ не приводится, поскольку оно является слишком громоздким).

Благодарности. Работа была частично поддержана РФФИ (грант № 05-0100126).

\section{Список литературы}

[1] V. Novikov, M. Shifman, A. Vainstein, V. Zakharov, Phys. Lett. B, 166 (1985), 329.

[2] W. Siegel, Phys. Lett. B, 84 (1979), 193.

[3] O. V. Tarasov, V. A. Vladimirov, Phys. Lett. B, 96 (1980), 94; I. Jack, D. R. T. Jones, C. G. North, Nucl. Phys. B, 486 (1997), 479.

[4] A. A. Slavnov, TMФ, 23 (1975), 3; T. Bakeyev, A. Slavnov, Mod. Phys. Lett. A, 11 (1996), 1539.

[5] A. Soloshenko, K. Stepanyantz, Two-loop renormalization of $N=1$ supersymmetric electrodynamics, regularized by higher derivatives, hep-th/0203118; А. Солошенко, К. Степаньянц,

[6] А. Солошенко, К. Степаньянц, ТMФ, 140 (2004), 437; hep-th/0304083.

[7] К. В. Степаньянц, TMФ, $142(2005), 37$.

[8] К.В. Степаньянц, TMФ, 146 (2006), 385.

[9] П. Уэст, Введение в суперсимметрию и супергравитацию, Мир, М., 1989.

[10] А. А. Славнов, Л. Д. Фаддеев, Введение в квантовую теорию калибровочных полей, Наука, М., 1988.

[11] Е. А. Андрияш, А. Б. Пименов, К. В. Степаньянц, Вестник МГУ. Сер. физика, астрономия, 4 (2005), 7.

Поступила в редакцию 31.Х.2005 г., после доработки 1.XII.2005 г. 